\title{
Review of Research Paper: Development of a Performance Verification Test for USP Apparatus 4
}

Erika S. Stippler

United States Pharmacopeial Convention, Rockville, MD
E aton et al. (1) recently published a research paper in the online edition of Pharmaceutical Research. The topic of the paper was the development of a Performance Verification Test (PVT) for USP dissolution Apparatus 4. This research paper was issued by a team of USP staff members.

The authors of this research paper investigated the suitability of a tablet formulation containing salicylic acid as a candidate reference material for the PVT of USP Apparatus 4. Currently USP General Chapter Dissolution $<711>$ has no method or requirement to assess the integrity of the overall procedure for Apparatus 4. The overall procedure includes the apparatus, the analytical procedure, and the analyst. For the development of a meaningful PVT, the authors also consider-besides the repeatability, the reproducibility, and the ruggedness of the method-its sensitivity to changes in operational parameters to be critical. Moreover, the PVT should be able to demonstrate sensitivity to instrument parameters that cannot be assessed solely by other measurements.

For this study, the authors selected a nondisintegrating tablet formulation that contains salicylic acid as an analytical marker. Tests were performed with USP Apparatus 4 operated in a closed-loop setting, and the tablets were placed into the 12-mm flow-through cell between layers of glass beads so that the position of the formulation did not change over the entire testing time. A total of $1000 \mathrm{~mL}$ of phosphate buffer solution $\mathrm{pH} 6.8$ was used for each test, and the nominal flow rate was $8 \mathrm{~mL} /$ min. A design-of-experiment (DoE) approach was used to probe the effect of eight variables that were considered to have an effect on either the mean amount dissolved or on the standard deviation of six replicates. The variables selected were flow rate, temperature of the medium in the flow-through cell, deaeration of the medium, amount of glass beads, size of the glass beads, orientation of the tablet in the flow-through cell, analyst, and instrument manufacturer. Tests were performed by two analysts using six cells on two USP Apparatus 4 instruments with variables set at two levels. The analysts completed 38 runs and recorded the mean and standard deviation results. Analysts used DoE software to evaluate the contribution of each variable individually as well as by two-factor interaction regarding both the mean and the standard deviation.

The study results show that four variables were significant for mean amount dissolved: size of glass beads, temperature in the flow-through cell, flow rate, and level of deaeration. The size of the glass beads had the predominant effect. Regarding the standard deviation results, two variables, tablet orientation and deaeration level, were significant. Three variables (i.e., analyst, instrument manufacturer, and amount of glass beads) statistically had no effect on either of the two responses.

The authors concluded that the large effect of the size of the glass beads on the mean amount dissolved indicates that the distribution of the glass beads used may be critical to obtain consistent results. The results also show that a PVT can be developed to ensure the integrity of the dissolution procedure with USP Apparatus 4.

\section{REFERENCES}

1. Eaton, J.;Tran, D.; Hauck, W.W.; Stippler, E. S. Development of a Performance Verification Test for USP Apparatus 4. Pharm Res. [Online early access]. DOI: 10.1007/s11095-011-0559-6. Published Online: Aug 9, 2011.http://www.springerlink.com/content/ h721245206531508/fulltext.html (accessed Oct 18, 2011). 